\title{
A Low Cross-Polarization Smooth-Walled Horn with Improved Bandwidth
}

\author{
Lingzhen Zeng, Charles L. Bennett, David T. Chuss, and Edward J. Wollack
}

\begin{abstract}
Corrugated feed horns offer excellent beam symmetry, main beam efficiency, and crosspolar response over wide bandwidths, but can be challenging to fabricate. An easier-tomanufacture smooth-walled feed is explored that approximates these properties over a finite bandwidth. The design, optimization and measurement of a monotonically-profiled, smoothwalled scalar feedhorn with a diffraction-limited $\sim 7^{\circ}$ FWHM is presented. The feed was demonstrated to have low cross polarization $(<-30 \mathrm{~dB})$ across the frequency range 33-45 $\mathrm{GHz}$ (30\% fractional bandwidth). A return loss better than $-28 \mathrm{~dB}$ was measured across the band.
\end{abstract}

\section{Index Terms}

antenna, feed, horn, millimeter-wave, optimization.

\section{INTRODUCTION}

$\sqrt{ } \begin{aligned} & \mathrm{ANY} \text { precision microwave applications require feedhorns with symmetric } E \text { - and } \\ & H \text {-plane beam patterns that possess low sidelobes and cross-polarization control. }\end{aligned}$ A common approach to achieving these goals is a "scalar" feed, which has a beam response that is independent of azimuthal angle. Corrugated feeds [1] approximate this idealization by providing the appropriate boundary conditions for the $H E_{11}$ hybrid mode at the feed aperture. Alternatively, an approximation to a scalar feed can be obtained with a multimode feed design. One such "dual-mode" horn is the Potter horn [2]. In this implementation, an appropriate admixture of $T M_{11}$ is generated from the initial $T E_{11}$ mode using a concentric step discontinuity in the waveguide. The two modes are then phased to achieve the proper July $6,2009-3: 20 \mathrm{pm}$

DRAFT 
field distribution at the feed aperture using a length of waveguide. The length of the phasing section limits the bandwidth due to the dispersion between the modes. Lier [3] has reviewed the cross-polarization properties of dual-mode horn antennas for selected geometries. Other authors have produced variations on this basic design concept [4], [5]. Improvements in the bandwidth have been realized by decreasing the phase difference between the two modes by $2 \pi[6],[7]$.

To increase the bandwidth, it is possible to add multiple concentric step continuities with the appropriate modal phasing [8], [9]. A variation on this technique is to use several distinct linear tapers to generate the proper modal content and phasing [10], [11]. Operational bandwidths of $15-20 \%$ have been reported using such techniques. A related class of devices is realized by allowing the feedhorn profile to vary smoothly rather than in discrete steps. Examples of such smooth-walled feedhorns with $\sim 15 \%$ fractional bandwidths exist in the literature [12], [13].

In this work, we describe the design and optimization of a smooth-walled feed that has a $30 \%$ operational bandwidth, over which the cross-polarization response is better than -30 $\mathrm{dB}$. The optimization technique is described, and the performance of the feed is compared with other published dual-mode feedhorns. The feedhorn described here has a monotonic profile that allows it to be manufactured by progressively milling the profile using a set of custom tools.

\section{Smooth-Walled Feedhorn Optimization}

The performance of a feedhorn can be characterized by angle- and frequency-dependent quantities that include beam width, sidelobe response and cross-polarization. Quantities such as return loss and polarization isolation that only depend on frequency are also important considerations. All of these functions are dependent upon the shape of the feed profile. In 
the optimization approach described, a weighted penalty function is used to explore and optimize the relationship between the feed profile and the electromagnetic response.

\section{A. Beam Response Calculation}

The smooth-walled horn was approximated by a profile that consists of discrete waveguide sections, each of constant radius. With this approach, it was important to verify that each section is thin enough that the model is a valid approximation of the continuous profile. For profiles relevant to our design parameters, section lengths of $\Delta l \leq \lambda_{c} / 20$ were found to be sufficient by trial and error, where $\lambda_{c}$ is the cutoff wavelength of the input waveguide section. It is possible in principle to dynamically set the length of each section to optimize the approximation to the local curvature of the horn. This would increase the speed of the optimization; however, for simplicity, this detail was not implemented in our study.

For each trial feedhorn the angular response was calculated directly from the modal content at the feed aperture. This in turn was calculated as follows. The throat of the feedhorn was assumed to be excited by the circular waveguide $T E_{11}$ mode. The modal content of each successive section was then determined by matching the boundary conditions at each interface using the method of James [14]. The cylindrical symmetry of the feed limits the possible propagating modes to those with the same azimuthal functional form as $T E_{11}$ [15]. This azimuthal-dependence extends to the resulting beam patterns, allowing the full beam pattern to be calculated from the $E$ - and $H$-plane angular response. Ludwig's third definition [16] is employed in calculation of cross-polar response. We note that an additional consequence of the feedhorn symmetry is that to the extent that the $E$ - and $H$-planes are equal in both phase and amplitude, the cross-polarization is zero [17]. Changes in curvature in the feed profile can excite higher order modes (e.g., $T E_{12}$ and $T M_{12}$ ), the presence of which can potentially degrade the cross-polarization response of the horn. 


\section{B. Penalty Function}

We constructed a penalty function to optimize the antenna profile. The penalty function with normalized weights, $\alpha_{j}$, is written as

$$
\chi^{2}=\sum_{i=1}^{N} \sum_{j=1}^{M}\left(\alpha_{j} \Delta_{j}\left(f_{i}\right)^{2}\right)
$$

where $i$ sums over a discrete set of $(N)$ frequencies in the optimization frequency band, and $j$ sums over the number $(M)$ of discrete parameters one wishes to take into account for the optimization. In the parameter space considered, this function was minimized over the frequency range $1.25 f_{c}<f<1.71 f_{c}\left(\Delta f / f_{0}=0.3\right)$ to find the desired solution. Results reported here were obtained by restricting this penalty function to include only the crosspolarization and return loss with uniform weights $(M=2)$. Additional parameters were explored; however, they were found to be subdominant in producing the target result. These functions were evaluated at 13 equally-spaced frequency points in Equation 1. The explicit forms used for $\Delta_{1}(f)$ and $\Delta_{2}(f)$ are

$$
\begin{aligned}
& \Delta_{1}(f)= \begin{cases}X P(f)-X P_{0} & \text { if } X P(f)>X P_{0}, \\
0 & \text { if } X P(f) \leq X P_{0},\end{cases} \\
& \Delta_{2}(f)= \begin{cases}R L(f)-R L_{0} & \text { if } R L(f)>R L_{0}, \\
0 & \text { if } R L(f) \leq R L_{0},\end{cases}
\end{aligned}
$$

where $X P(f)$ and $R L(f)$ are the maximum of the cross-polarization $X P(f)=\operatorname{Max}[X P(f, \theta)]$ and return loss at frequency $f$, respectively. $X P_{0}$ and $R L_{0}$ are the threshold cross-polarization and return loss. If either the cross-polarization or return loss at a sampling frequency were less than its critical value, it was omitted from the penalty function. Otherwise, its squared difference was included in the sum in Equation 1. 


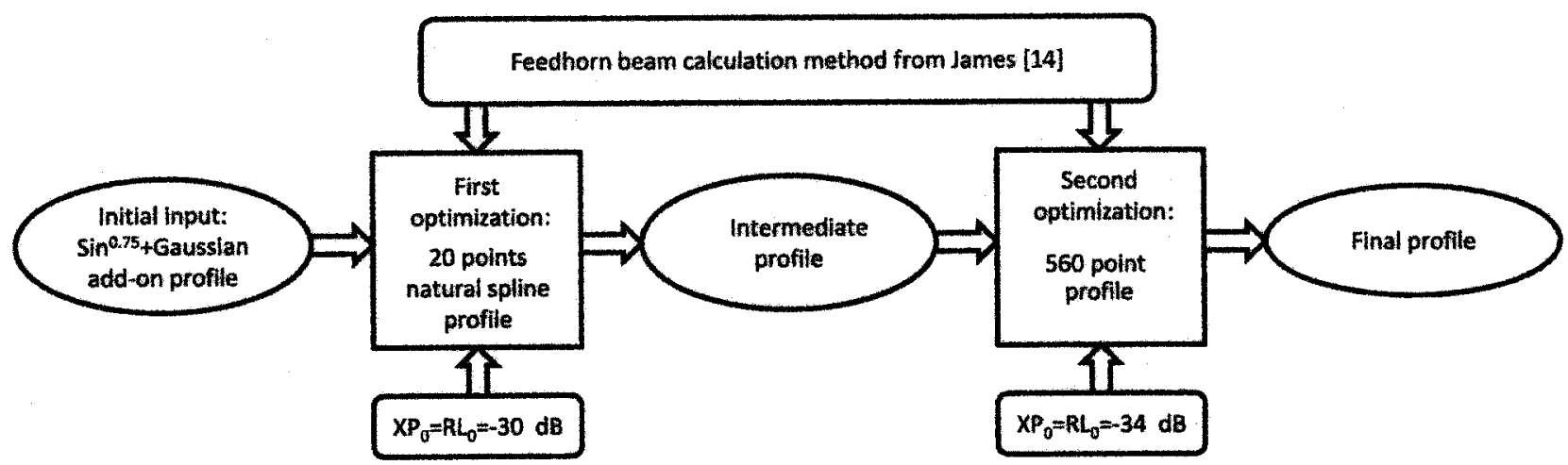

Fig. 1. The feedhorn profile was represented by a series of thin, concentric waveguides. The modal content and corresponding beam properties were calculated by matching boundary conditions at the waveguide interfaces. At each stage of optimization, the penalty function defined in Equation 1 was minimized. The first stage optimization was done using a 20-point natural spline parameterization; during the second stage each section of the 560-point approximation of the feedhorn was permitted to vary in radius.

\section{Feedhorn Optimization}

As shown in Figure 1, the feedhorn was optimized in a two-stage process that employed a variant of Powell's method [18]. Powell's method is a rapidly-converging method for finding the minima of a multi-variable function without explicit analytical expressions for its partial derivatives. In this method, every variable of the function is varied during the optimization. Generically, this algorithm can produce an arbitrary profile. To produce a feed that is easily machinable, we restricted the optimization to the subset of profiles for which the radius increases monotonically along the length of the horn. Without this constraint, this method was observed to explore solutions with corrugated features and the serpentine profiles explored in [19].

The aperture diameter of the feedhorn was initially set to $\sim 4 \lambda_{c}$, but was allowed to vary slightly to achieve the desired beam size. A single discontinuity exists between the circular waveguide and the feed throat. The remainder of the horn profile adiabatically transitions to the feed aperture. The total length of the feedhorn from the aperture to the single mode waveguide was fixed at $12.3 \lambda_{c}$ during optimization. This length is somewhat arbitrary, but chosen to produce a diffraction-limited beam in a practical volume. 
The approach of [12] was followed as an initial input to the Powell method. Specifically, the feed radius, $r$, is written analytically as a function of the distance along the length of the horn, $z$, as:

$$
r(z)= \begin{cases}0.293+0.703 \sin ^{0.75}(0.255 z) & 0 \leq z \leq 6.15 \\ 0.293+0.703\left\{1+[0.282(z-6.15)]^{2}\right\}^{1 / 2} & 6.15<z \leq 12.30\end{cases}
$$

where parameters are given in units of $\lambda_{c}$. This profile was then approximated by natural spline of a set of 20 points equally-spaced along the feed length. Throughout the optimization, we explicitly imposed the condition that radius of each section be greater than or equal to that of the previous section. This sampling choice effectively limits the allowed change in curvature along the feed profile. In the first stage of optimization, both $X P_{0}$ and $R L_{0}$ were set to $-30 \mathrm{~dB}$. The minimum of the penalty function was found by the modified Powell method in this 20-dimension space.

In the second stage of the optimization, the number of points explicitly varied along the profile was increased to 560. The modified Powell method was used to optimize the profile in this 560-dimensional space. In this phase, both of $X P_{0}$ and $R L_{0}$ were decreased to -34 dB.

In principle, it is possible to use either of these techniques alone to find our solution. There are enough degrees of freedom in the 20-point spline to do so and the 560-point technique should be able to recover the solution regardless of the starting point. We found, however, that the 20-point spline did not converge readily to the final profile given the initial conditions above, but rather converged to a broad local minimum. In addition to finding the general features of the desired performance, this first phase of optimization provided a significant reduction in the use of computing resources compared to the slower 560-point parameter search. 


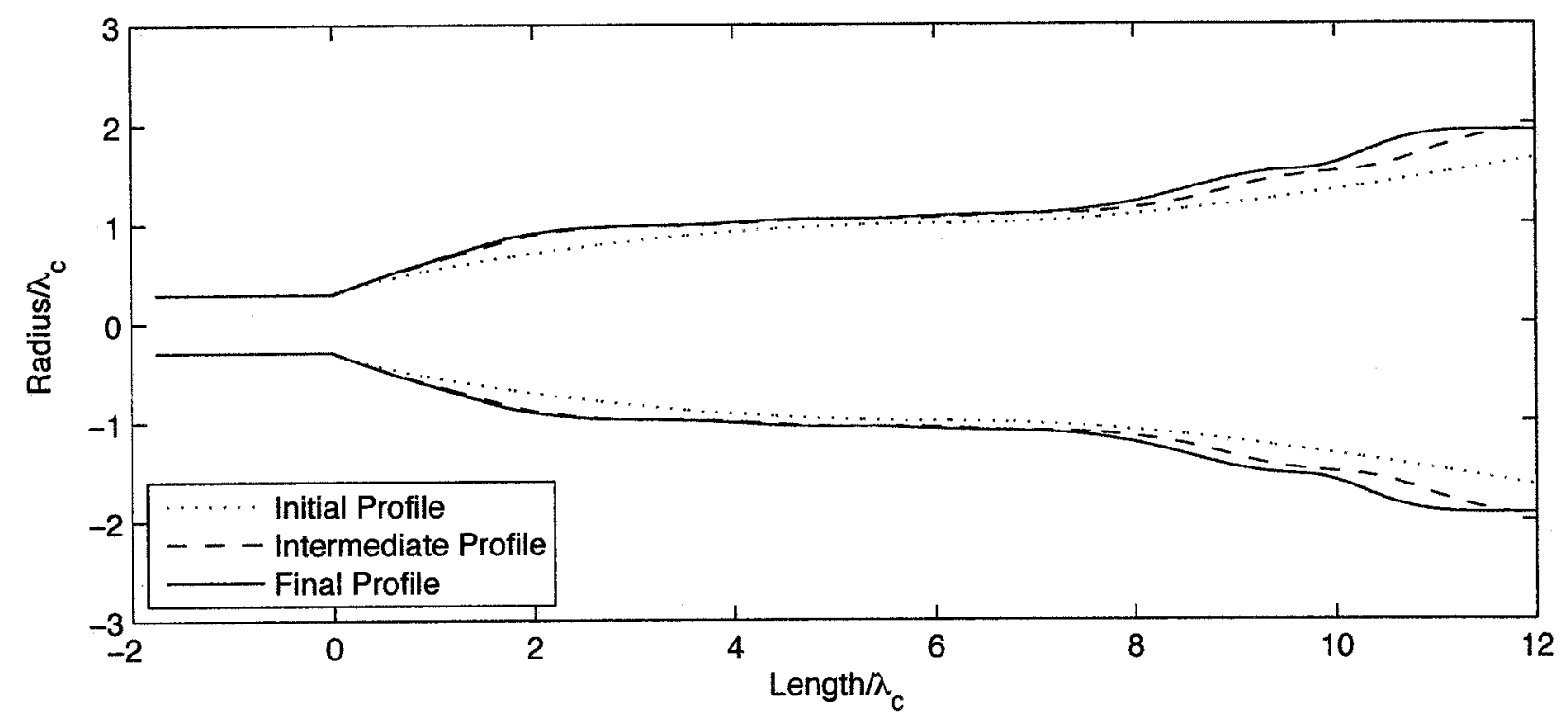

Fig. 2. The initial, intermediate and final profiles are shown. All dimensions are given in units of the cuttoff wavelength of the input circular waveguide.

Figure 2 shows the initial, intermediate, and final feedhorn profiles. It is possible to approximate the final profile with a 20-point spline. The final profile of the feed is reproduced with a low-spatial frequency error of $\sim 0.015 \lambda_{c}$. This effect has a negligible influence on the modeled performance. This suggests that the optimization procedure could be done completely using a spline with fewer than 20 points if the location of the spline points were dynamically varied. Future optimization algorithms could be made more efficient by implementing this approach. Figure 3 shows the improvement in cross-polarization for the two stages of optimization. The return loss is also shown for the initial profile, the intermediate optimization, and the final feedhorn profile.

\section{iII. FeEdhorn FAbrication and MEASUREment}

A feed (Figure 4) that operates in circular waveguide with a $T E_{11}$ cutoff frequency of $f_{c}=26.36 \mathrm{GHz}$ was fabricated to test the proposed design. The structure was optimized between 33 and $45 \mathrm{GHz}$. The prototype feed was manufactured via electroforming in order July $6,2009-3: 20 \mathrm{pm}$

DRAFT 


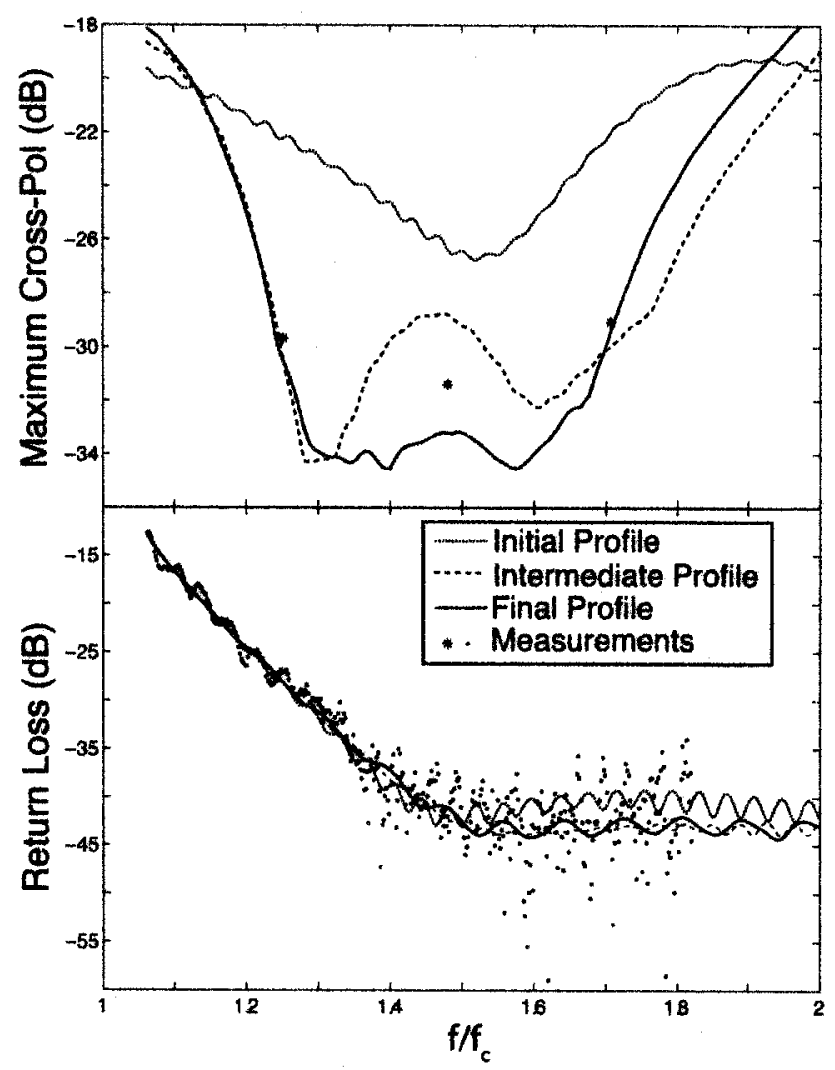

Fig. 3. (Top) The maximum cross-polar response across the band is shown for the three profiles in Figure 2. Measurements of the maximum cross-polarization are superposed. (Bottom) The return loss measurements for the final feed horn are shown plotted over the predicted return loss for the initial, intermediate, and final feedhorn profiles. Frequency is given in units of the cutoff frequency of the input circular waveguide.

to validate the design using a process that allows the feed structure to be measured and compared to the design profile. The final design profile is well-approximated by splining the radius $(r)$ as a function of length $(z)$ provided in Table I. The full 560-point profile is available upon request.

The feedhorn was measured in the Goddard Electromagnetic Anechoic Chamber (GEMAC). The receivers and microwave wave sources used in the measurement provide $\mathrm{a}>50 \mathrm{~dB} d \mathrm{~d}-$ namic range from the peak response over $\sim 2 \pi$ steradians with an absolute accuracy of $<0.5 \mathrm{~dB}$. A five section constant cutoff transition from rectangular waveguide (WR 22.4, $f_{c}=26.36 \mathrm{GHz}$ ) to circular waveguide [20] was used to mate the feedhorn to the rectangular 
waveguide of the antenna range infrastructure. The constant cutoff condition was maintained in the transition by ensuring $a_{\text {circle }}=a_{\text {broadwall }} s_{11} / \pi$ where $a_{\text {circle }}$ is the radius of the circular guide, $a_{\text {broadwall }}$ is the width of the broadwall of the rectangular guide, and $s_{11} \sim 1.841$ is the eigenvalue for the $T E_{11}$ mode [21]. The alignment of the circular waveguide feed interface was maintained to avoid degradation of the cross-polar antenna response. Pinning of this interface as specified in [22] or similar is recommended. Beam plots and parameters at the extrema and the middle of the optimization frequency range are shown in Figure 5 and Table II. The cross-polarization response as a function of frequency of this device is compared to other published implementations of multi-mode scalar feeds (Fig. 6). An HP8510C network analzyer was used to measure the return loss (see Fig. 3) with a through-reflect-line calibration in circular waveguide. If desired, the match at the lower band edge can be improved by using a transition to a larger diameter guide. The measured observations are in agreement with theory.

Imperfections in the profile may occur during manufacturing due to chattering of the tooling or similar physical processes. We performed a tolerance study to determine the effect of such high-spatial frequency errors in the feed radius. Negligible degradation in performance was observed for Gaussian errors in the radius up to $0.002 \lambda_{c}$. The feed's monotonic profile is compatible with machining by progressive plunge milling in which successively more accurate tools are used to realize the feed profile. This technique has been used for individual feeds and is potentially useful for fabricating large arrays of feedhorns. Examples include, fabrication of multimode Winston concentrators [23], [24], direct-machined smooth-walled conical feed horns for the South Pole Telescope [25], and the exploration of this techneque for dual-mode feedhorns [10]. 
TABLE I

SPLINE APPROXIMATION TO OPTIMIZED PROFILE (IN MILLIMETERS)

\begin{tabular}{ccc}
\hline Section & Length $(z)$ & Radius $(r)$ \\
\hline 0 & 0.0 & 3.334 \\
1 & 7.0 & 5.768 \\
2 & 14.0 & 7.909 \\
3 & 21.0 & 9.901 \\
4 & 28.0 & 10.858 \\
5 & 35.0 & 11.131 \\
6 & 42.0 & 11.269 \\
7 & 49.0 & 11.663 \\
8 & 56.0 & 11.903 \\
9 & 63.0 & 11.957 \\
10 & 70.0 & 12.238 \\
11 & 77.0 & 12.442 \\
12 & 84.0 & 12.760 \\
13 & 91.0 & 13.704 \\
14 & 98.0 & 15.399 \\
15 & 105.0 & 17.012 \\
16 & 112.0 & 17.706 \\
17 & 119.0 & 20.054 \\
18 & 126.0 & 21.747 \\
19 & 133.0 & 21.914 \\
20 & 140.0 & 21.916 \\
\hline
\end{tabular}

TABLE II

BEAM PARAMETERS

\begin{tabular}{cccc}
$\begin{array}{c}\text { Frequency } \\
{[\mathrm{GHz}]}\end{array}$ & $\begin{array}{c}\text { Wavelength } \\
{[\mathrm{mm}]}\end{array}$ & $\begin{array}{c}\text { Antenna Gain } \\
{[\mathrm{dBi}]}\end{array}$ & $\begin{array}{c}\text { Beam Solid Angle } \\
{[\mathrm{Sr}]}\end{array}$ \\
\hline & & & \\
33 & 9.09 & 21.3 & 0.0925 \\
39 & 7.69 & 22.0 & 0.0788 \\
45 & 6.67 & 24.2 & 0.0473 \\
\hline
\end{tabular}

IV. CONCLUSION

An optimization technique for a smooth-walled scalar feedhorn has been presented. Using this flexible approach, we have demonstrated a design having a $30 \%$ bandwidth with cross-polar response below $-30 \mathrm{~dB}$. The design was tested in the range $33-45 \mathrm{GHz}$ and found to be in agreement with theory. The design's monotonic profile and tolerance insensitivity 


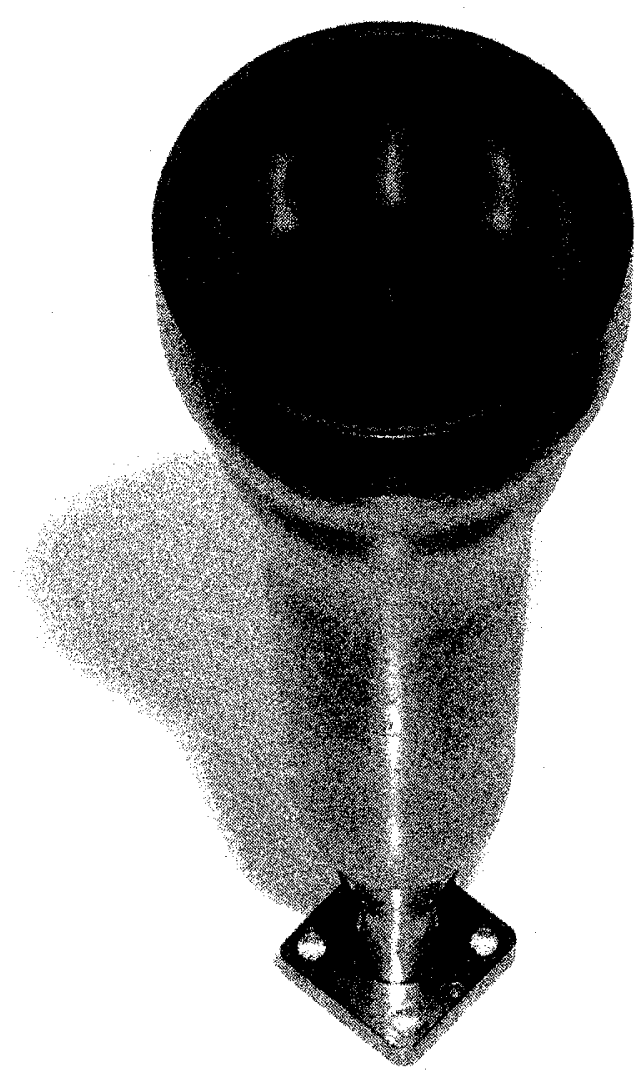

Fig. 4. A smooth-walled feedhorn operating between 33 and $45 \mathrm{GHz}$ was constructed. The horn is $140 \mathrm{~mm}$ long with an aperture radius of $22 \mathrm{~mm}$. The input circular waveguide radius is $3.334 \mathrm{~mm}$.

enable the manufacturing of such feeds by direct machining. This approach is useful in applications where a large number of feeds are desired in a planar array format.

\section{ACKNOWLEDGEMENTS}

We thank Harvey Moseley for his support of this work. We thank Steve Seufert and Ken Hersey for their help with the measurements of the prototype. We thank Dale Fixsen for useful discussions. This work was funded in part by a NASA ROSES APRA grant and a Johns Hopkins University/APL partnership research grant. 


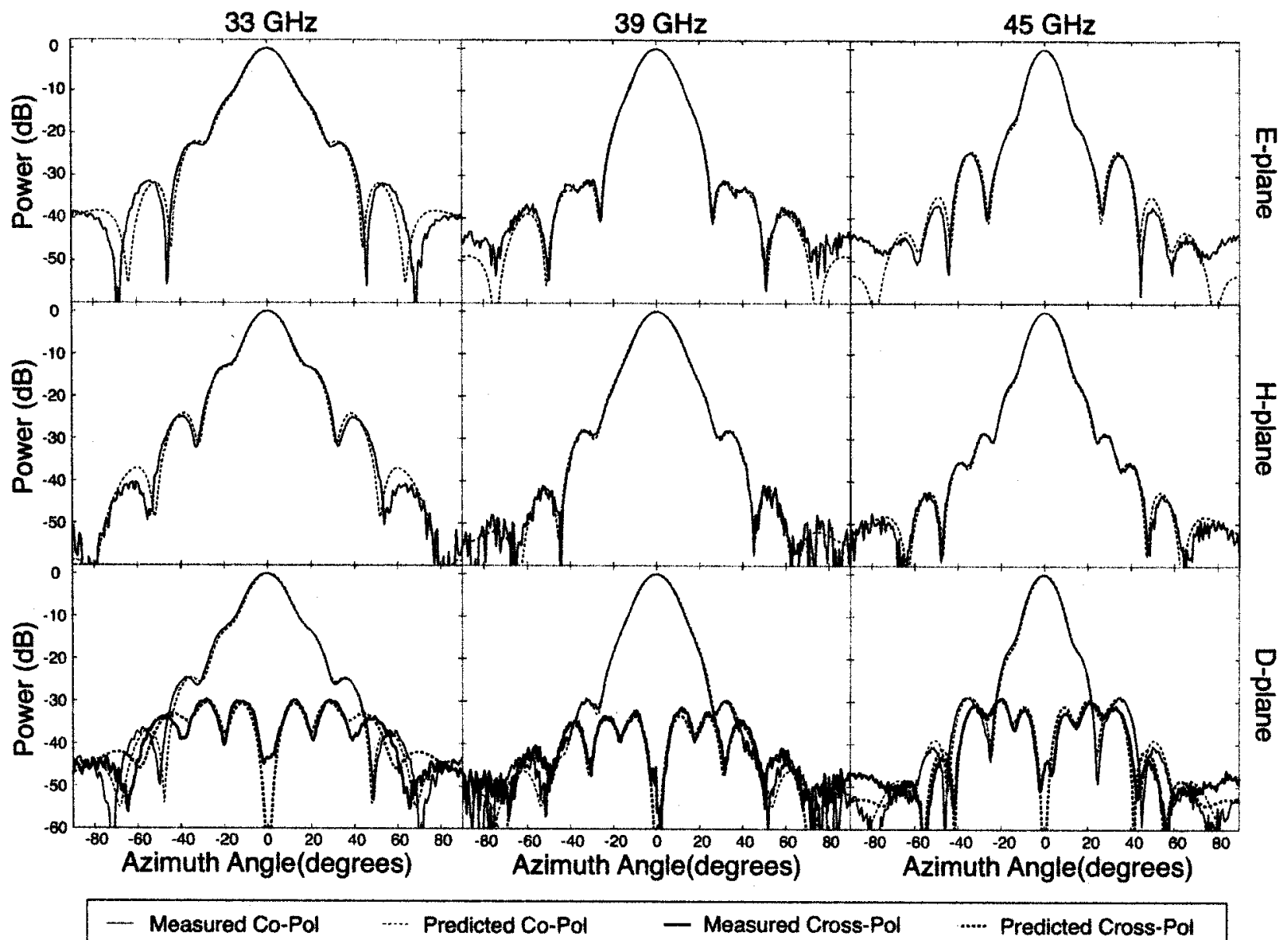

Fig. 5. The measured $E-, H$-, and diagonal-plane angular responses for the lower edge ( $33 \mathrm{GHz})$, center $(39 \mathrm{GHz})$, and upper edge $(45 \mathrm{GHz})$ of the optimization band are shown. The cross-polar patterns in the diagonal plane are shown in the bottom three panels for each of the three frequencies.

\section{REFERENCES}

[1] P. Clarricoats and A. Olver, Corrugated Horns for Microwave Antennas. Peter Peregrinus, Ltd., 1984.

[2] P. Potter, "A new horn antenna with suppressed sidelobes and equal beamwidths," Microwave Journal, pp. 71-78, June 1963.

[3] E. Lier, "Cross polarization from dual mode horn antennas," IEEE Transactions on Antennas and Propagation, vol. AP-34, no. 1, pp. 106-110, 1986.

[4] R. Turrin, "Dual mode small-aperture antennas," IEEE Transactions on Antennas and DRAFT 


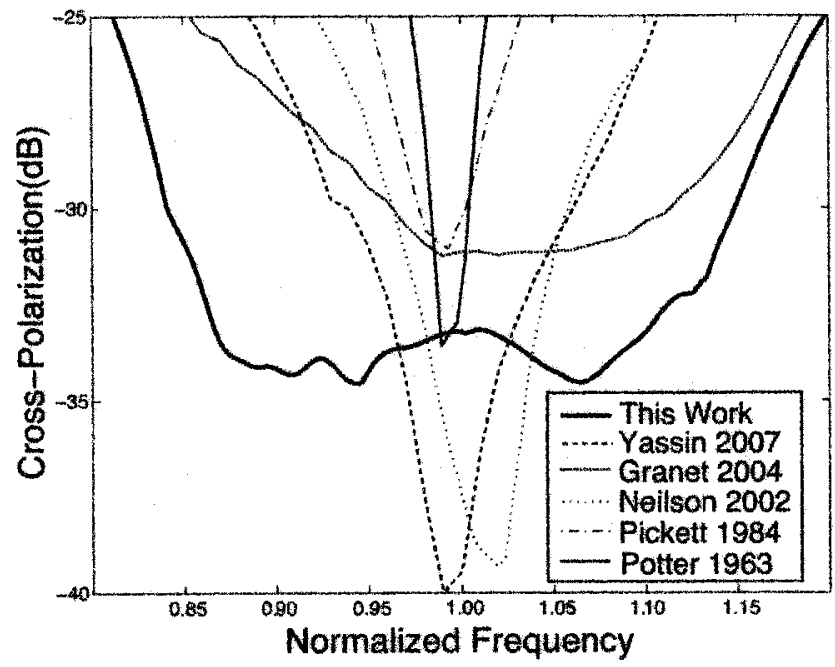

Fig. 6. The maximum cross-polar response of the prototype feedhorn is compared to other implementations of smooth-walled feedhorns. The data presented have been normalized to the design center frequencies as specified by the respective authors.

Propagation, vol. 15, no. 2, pp. 307 - 308, Jan 1967.

[5] G. Ediss, "Technical memorandum. dual-mode horns at millimetre and submillimetre wavelengths," IEE Proceedings H Microwaves Antennas and Propagation, vol. 132, no. 3, pp. $215-218$, Jun 1985.

[6] H. Pickett, J. Hardy, and J. Farhoomand, "Characterization of a dual-mode horn for submillimeter wavelengths (short papers)," IEEE Transactions on Microwave Theory and Techniques, vol. 32, no. 8, pp. 936 - 937, Jan 1984.

[7] S. Skobelev, B.-J. Ku, A. Shishlov, and D.-S. Ahn, "Optimum geometry and performance of a dual-mode horn modification," IEEE Antennas and Propagation Magazine, vol. 43, no. 1, pp. 90-93, Mar 2001.

[8] T. Bird, "A multibeam feed for the parkes radio-telescope," IEEE Antennas \& Propagation Symposium, pp. 966--969, Mar 1994.

[9] S. M. Tun and P. Foster, "Computer optimised wideband and dual-mode horn," Electronics Letters, vol. 38, no. 15, pp. 768-769, Jul 2001. 
[10] G. Yassin, P. Kittara, A. Wangsuya, S. Jiralucksanawong, J. Leech, and M. Jones, "A high performance horn for large format focal plane arrays," 18th International Symposium on Space Terahertz Technology, pp. 1-12, Apr 2008.

[11] P. Kittara, A. Jiralucksanawong, G. Yassin, S. Wangsuya, and J. Leech, "The design of potter horns for thz applications using a genetic algorithm," International Journal of Infrared and Millimeter Waves, vol. 28, p. 1103, Dec 2007, (c) 2007: Springer Science+Business Media, LLC.

[12] G. Granet and G. James, "A smooth-walled spline-profile horn as an alternative to the corrugated horn for wide band millimeter-wave applications," IEEE Transactions on Antennas and Propagation, vol. 52, no. 3, pp. 848-854, Apr 2004.

[13] J. Neilson, "An improved multimode horn for gaussian mode generation at millimeter and submillimeter wavelengths," IEEE Transactions on Antennas and Propagation, vol. 50, no. 8, pp. 1077-1081, Jul 2002.

[14] G. James, "Analysis and design of TE-11 to HE-11 corrugated cylindrical waveguide mode converters," IEEE Transactions on Microwave Theory and Techniques, vol. MTT29, no. 10, pp. $1059-1066$, Oct 1981.

[15] A. Olvers, P. Clarricoates, A. Kishk, and L. Shafai, Microwave Horns and Feeds. IEEE Press, 1994.

[16] A. Ludwig, "The definition of cross polarization," IEEE Transactions on Antennas and Propagation, vol. 21, no. 1, pp. 116 - 119, Jan 1973.

[17] P.-S. Kildal, Foundations of Antennas: A Unified Approach. Studentlitteratur, 2000.

[18] W. Press, S. Teukolsky, W. Vetterling, and B. Flannery, Numerical Recipes in C, 2nd ed. Cambridge University Press, 1992.

[19] H. Deguchi, M. Tsuji, and H. Shigesawa, "Compact low-cross-polarization horn antenDRAFT July $6,2009-3: 20 \mathrm{pm}$ 
nas with serpentine-shaped taper," IEEE Transactions on Antennas and Propagation, vol. 52 , no. 10 , pp. 2510-2516, Sep 2004.

[20] E. Wollack, "TCHEB_x: Homogeneous stepped waveguide transformers," NRAO, EDTN Memo Series \# 176, May 1996.

[21] J. Pyle and R. Angley, "Cutoff wavelengths of waveguides with unusual cross sections (correspondence)," IEEE Transactions on Microwave Theory and Techniques, vol. 12, no. 5, pp. $556-557$, Jan 1964 .

[22] J. Hesler, A. Kerr, W. Grammer, and E. Wollack, "Recommendations for waveguide interfaces and frequency bands to $1 \mathrm{THz}, " 18$ th International Symposium on Space Terahertz Technology, pp. 100-103, 2007.

[23] D. J. Fixsen, "Multimode antenna optimization," R. Winston, Ed., vol. 4446, no. 1. SPIE, 2001, pp. 161-170.

[24] D. J. Fixsen, E. S. Cheng, T. M. Crawford, S. S. Meyer, G. W. Wilson, E. S. Oh, and E. H. S. III, "Lightweight long-hold-time dewar," Review of Scientific Instruments, vol. 72 , no. 7 , pp. $3112-3120,2001$.

[25] W. Holzapfel and J. Ruhl, Private Communication, 2009.

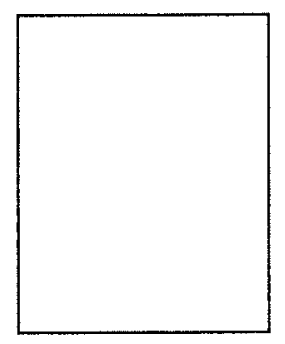

\section{Lingzhen Zeng}




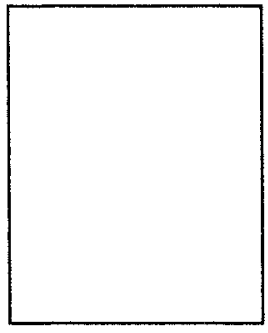

Charles L. Bennett

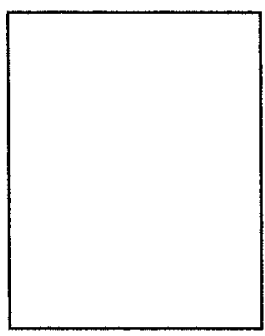

David T. Chuss

Edward J. Wollack 